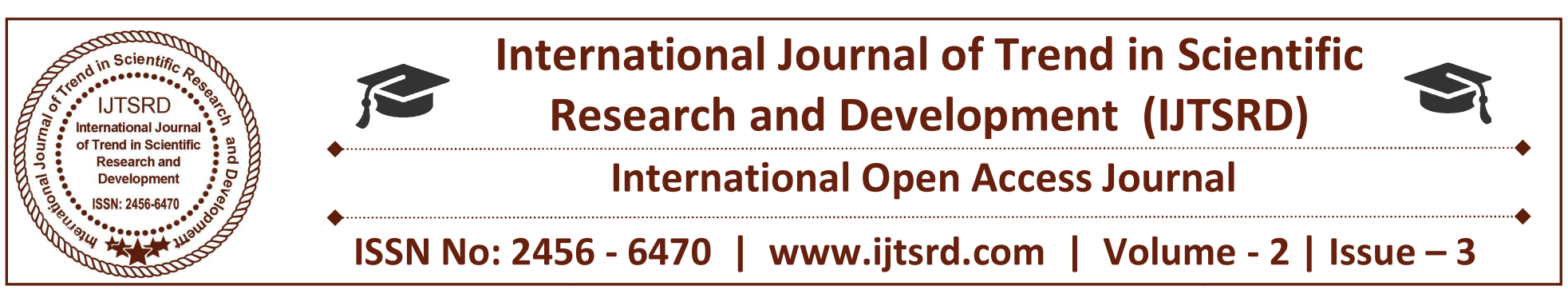

\title{
Performance through Efficient Robust Routing Technique (ERR Model) to Protect WSN from DoS Attack
}

\author{
Bablu Kumar Mishra \\ M.Tech Scholar, Department of Computer \\ Science \& Engineering, Sagar Institute of \\ Research \& Technology, Bhopal, India
}

\author{
Sunil Malviya \\ Assistant Professor, Department of Computer \\ Science \& Engineering, Sagar Institute of \\ Research \& Technology, Bhopal, India
}

\section{ABSTRACT}

In the Era of such a growing communication environment, everyone are now use to of wireless sensor network, sensor based various research has been going on to make WSN more reliable, secure and efficient for the betterment of digital communication services, since digital data are growing in day to day life it does more traffic and need high quality communication network so that one can rely on them in the similar journey one has analyzed with various literature and communication services provided by SAODV and RAEED-EA protocols specially designed for WSN services which has been immune the WSN to DoS attack, since WSN consists of various sensor and non sensor based communication devices in the form of small network nodes with sensing capability, power consumption and management techniques along with energy efficiency based protocols and nodes to control and protect over the WSN. One analyzed the service scenario of traditional and latest protocols designed for better communication service environment proposed model will make WSN to be error free from physical and Data link layer errors and attacks many research are doing research, in the same way in this thesis we are try to overcome the drawback of WSN routing at physical and Data link layer to through our proposed model integrate an efficient routing algorithm that improve performance of routing over tradition WSN routing protocol along with this proposed ERR model also immune the WSN from physical and Data link layer attacks (Jamming, collision) issues so that reliability can be achieve.
Keywords: WSN, DoS Attack, ERR Model, SAODV, $R A E E D-A$

\section{INTRODUCTION}

At this time WSN (Wireless Sensor Network) is the most usual services in work in commercial and trade applications, because of its procedural development in a workstation, communication, and low-power usage of surrounded compute procedure. The WSN build with nodes that are used to monitor the environment like high temperature, moisture, stress, point, trembling, noise etc.

These nodes can use in different genuine-time applications to execute different everyday jobs like smart detecting, a finding of neighbor node, information dispensation and storage space, data gathering, objective track, examine and controlling. .

Wireless Sensor networks (WSNs) rely on wireless message, which is a type of distribution medium and susceptible to be eavesdropped [1]. The adversary may exercise luxurious telephone lines transceivers to cooperate with the network and to perceive the meaning, and then draw back to the communication source by stirring all along the upturned path [4], even if well-built data encryption is utilize. The entity, e.g., the endanger animal variety, or a vehicle of forces aide, may have to be confined for shelter reason and the correlated place in sequence should not be disclose. This distress will grow to be level more stern for prospect antenna network frequency in constant compute applications, as the in all places information collections doubtlessly encroach on the isolation of 
the public complicated [3]. a lot of technique to talk to the cause position isolation concern have been projected, see [4],where specter routing is one of the in style approaches for preserve WSN as per the need of its user.

\section{RELATED WORK}

The privacy from the various problems related to threats that be present in wireless sensor networks largely classify along two proportions likely (i) substance-based confidentiality threats and (ii) circumstance-based confidentiality threats [4]. While substance-based confidentiality threats are well silent [1], with cryptographic technique frequently being use to attend to these issue [9], cryptographic methods do not find circumstance-based confidentiality threats and circumstance-based confidentiality threats has challenge [10]. One significant feature of circumstance-based confidentiality threats in several applications is resource protection. There are various research of sender location security protection in wireless sensor networks [5] and the previous study works can be divided into different parts based on hackers skills, called node security preservation protocol for maintaining local nodes attacks [6], and resources protection security preservation protocol for universal node attacks [1]. In order to get protection universal node attacker against to universal node monitoring in [9] proposed WSN Rate protocol, in which no matter whether the original information is received, all nodes in the entire network from WSN will transmit data signals with specific rate of transmission. This protocol efficiently works against the universal node traffic analysis from different attacks.

As an extension, a local network WSN protocol is proposed in [2], where few sensor host works as local can have out duplicate data, so that it decreasing network load to some scope. In [6] proposed new Rate protocol. By defining the node based data transmission rate, the source address privacy can be preserved protected and the manage delay can be also compact. Authors in [9] introduced a new scheme called Optimal Filtering protocols communication Scheme to increase the network capacity to manage node and its life time to get preserve against universal hackers or attackers. However, for the universal hackers or attackers, in [8] have limitations. Since all host systems are transmitting a large scale fake or duplicate packets, which would increase the energy consumption and utilization of nodes as result also down reduce the WS network life, but also get prospect of data congestion and down the effectiveness of packet spreading. It is still a big problem and lots of research has been going on.

\section{LITERATURE SURVEY}

In [3] An Evolutionary method to get betters the capacity of the Wireless sensor network". Here one worked on the technique that how power effectiveness in the wireless sensor network is amplified by inherent algorithm scheme? Inherent algorithm scheme are practical in such a way to diminish the unnecessary data to the go down and protect its power funds, thereby, growing the capacity of nodes [3]. In [4], study was done, how a complete network patterns can be made for system delay in university grounds environment. The network interruption measures base on broadcast interruption and transmission delay. This network pattern can use to assess and examine network delay performance for research and preparation purposes. In [6], model of a multi-node self-organize wireless antenna set-up has been discuss. The idea of the implementation was to inspect, the presentation of self-connected wireless network design. In this way, such parameters as system configuration time, data packet transmission routine, throughput, and tool responsibility series have been examine. In addition, the contact of access protocols. In [7], various types of map-reading protocols are discussed. Where channel level communication, modifiable code and path fix were implementing and evaluate. Connection level regeneration manages link break and disputation very proficiently. Modifiable code introduces fixed cost. path fix immune by repeated losses, increasing convenience of modifiable code. In [8], routing protocols related with the cost relevant with energy that has been not used at each node of data transfer was detailed as earlier. They terminate that when only the communication energy is careful as the cost required, by means of shorter multi-hop relations seem to be more beneficial. In [9] the accessibility of wireless nodes is measured by the prism of node planning. They have checked the framework of sensor nodes that go with distance in - the area of testing parts which is must for raising the accessibility of WSNs. with COTS specifications tested system test interfaces for distance node testing, restore and software improve. The framework provided most favorable utilization. 
In [10] they have work on WSN nodes and assemble them in cellular arrangement, to optimize the reporting area, consistency in

Getting in sequence from the nodes and minimize beating of information's are enhanced. These design arrangements provide current explanation to sanctuary vital and forces applications [2].

\section{ERR ALGORITHM}

- Initialized all the Nodes, Shield Nodes, Target node (Original Server), along with its initial values.

- Now enabled Shield server nodes to get accept and reject connections with original server node.

- Maintain cache node to manage wait for connection states. For all the upcoming and current connection requests.

- To verify connection authentication perform auto authentication process via shield nodes to check:

- Whether Source exceeds its connection limit? If yes then it reject the connection request since it may be DoS client, otherwise go to next.

- Check whether Source exceeds its bandwidth limit? If yes then again the shield node will reject the connection request since it may be DoS client otherwise the request has been accepted for further connection process and data accessing permissions.

- Client would be acknowledged and accepted for the connection with original target server.

- Connection established.

- Receive server response.

In this way a shield based routing techniques gets best favorable solution to us to get opt the routing efficient and also get perceive the network from the major error oriented networking situation that defines how the network grow as high as possible along with the security concern and specification for the purpose of routing at WSN.

\section{OBJECTIVES AND FLOW OF ERR}

- WSN is a very challenging area of networking, many researchers is working on the problem statements, in this thesis we try to achieve following objectives.
- To simulate denial of service attacks against an Internet web server; Then to successfully defend against those attacks.

- Proposed ERR model will focus on the errors raised at physical layer during communication like to avoid Jamming Attack to maintain the performance and reliability of WSN.

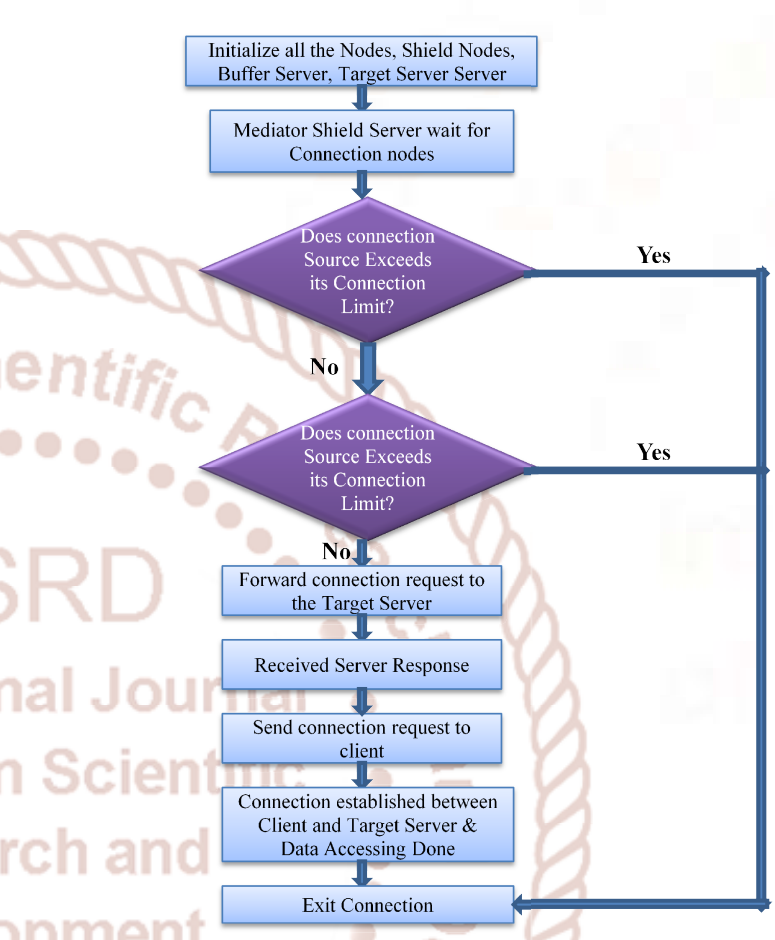

Fig.1 Flow chart of Efficient Robust Routing Technique in WSN

\section{CONCLUSION}

In the beginning of this paper we explain about the proposed technique in WSN along with we discussed the introduction of our proposed model, in further section we define related work and traditional methods used towards to get the objectives, in the next section we go through the literature analysis, objectives and proposed algorithm with flow chart through which we are trying to develop and implement an Efficient WSN algorithm to overcome from the problem occurred via DoS attack in WSN. At the end we can say the implementation module proposed by us will find better result compare to the previous techniques and algorithm. 


\section{REFERENCES}

1. Khan, A., Sensors Lifetime Enhancement Techniques in Wireless SensorNetworks - A Survey Journal of Computing, vol. 2, issue 5, May 2010.

2. Bachir, A., Dohler, M., Watteyne, T., Leung, K., MAC Essentials for Wireless Sensor Networks. Communications Surveys \& Tutorials, IEEE. Vol. 12, issue2, 2012 pp. 222-248.

3. Y. Yan, Y. Qian, H. Sharif, and D. Tipper, "A survey on smart grid communication infrastructures: Motivations, requirements and challenges," IEEE Commun. Surveys Tuts., vol. 15, no. 1, pp. 5_20,1st Quart., 2013.

4. Hui Jiang Energy big data: A survey, IEEE Journal and Magzine, vol. 4, 2016, pp 38443861DOI: 10.1109/ACCESS.2016.2580581.

5. Adam B. published "Structural Health Monitoring Using Wireless Sensor Networks: A Comprehensive Survey in IEEE Communications Surveys \& Tutorials, VOL. 19, and NO. 3, Third Quarter 2017.

6. Muhammad Asif1, Shafiullah Khan," Quality of Service of Routing Protocols in Wireless Sensor Networks: A Review" vol. 5, 2017, pp 1846-1871, DOI: $10.1109 /$ ACCESS.2017.2654356

7. Sunil Kumar Singh," A Survey On Successors Of Leach Protocol" IEEE Access vol. 5, 2017, pp 4298-4328,DOI:10.1109/ACCESS.2017.2666082.

8. Victoria J. Hodge,"Wireless Sensor Networks for Condition Monitoring in the Railway Industry: A Survey"IEEE Transactions on Intelligent Transportation Systems, Vol. 16, No. 3, June 2015.

9. Hlabishi I. Kobo,” A Survey On Software-Defined Wireless Sensor Networks: Challenges And Design Requirements" vol. 5, 2017, pp 1872 1899, DOI:10.1109/ACCESS.2017.2666200.

10. Nikos Bizanis ,Fernando A. Kuipers," SDN And Virtualization Solutions For The Internet Of Things: A Survey" vol. 4, 2016, pp 5591-5606, DOI: 10.1109/ACCESS.2016.2607786. 